\title{
Factores humanos y seguridad del personal de salud en tiempos de pandemia
}

\author{
Human factors and healthcare personnel \\ safety in times of pandemic
}

\author{
Diego Andrés Díaz-Guio, ${ }^{*, \ddagger}$ Elena Ríos-Barrientos, ${ }^{\S}$ \\ Pablo Andrés Santillán-Roldán," Viviana Medina, " Diego Fernando Salazar-Ocampo,*,** \\ Bonifacio Cimadevilla-Calvo, ${ }^{\ddagger}$ Alejandra Ricardo-Zapata*
}

Palabras clave: COVID-19, SARSCoV-2, habilidades no técnicas, carga cognitiva, equipo de protección personal.

Keywords: COVID-19, SARS$\mathrm{CoV}-2$, non-technical skills, cognitive load, personal protective equipment.

* Grupo de Investigación en Educación y

Simulación (EdSimC), Centro de Simulación Clínica VitalCare.

Armenia, Colombia.

¥ Doctorado en

Educación, Facultad de

Artes y Humanidades,

Universidad de Caldas.

Manizales, Colombia.

Servicio de Medicina

Crítica y Cuidados

Intensivos, Hospital

Universitario San Juan de

Dios. Armenia, Colombia.

$\S$ Centro de Simulación

Clínica, Tecnológico de

Monterrey, Escuela de

Medicina y Ciencias de la

Salud. Monterrey, México.

Recibido: 17/07/2020

Aceptado: 11/08/2020

doi: $10.35366 / 95232$

\section{RESUMEN}

El riesgo de contagio para los equipos de salud que atienden en primera línea la pandemia por COVID-19 es una realidad preocupante, más aún en Latinoamérica, el nuevo epicentro, donde convergen las condiciones de la enfermedad y la problemática social propia de una región de ingresos medios y bajos. Podremos pensar que disponer de equipos de protección personal de alta calidad es suficiente garantía para estar seguros y la evidencia nos demuestra que no es el único componente involucrado en la protección o el riesgo; como en la mayoría de las crisis, los factores humanos o habilidades no técnicas pueden ser determinantes de protección cuando se dominan o de riesgo cuando no se gestionan apropiadamente, dentro de la dinámica de trabajo en equipo. Los centros de simulación tienen la capacidad de participar en la construcción de equipos clínicos de alto rendimiento, que puedan enfrentar con mayor seguridad a este nuevo enemigo, que amenaza por igual a pacientes y personal de salud.

\section{ABSTRACT}

The contagion risk for the healthcare teams that attend the pandemic at the frontlines is a worrying reality, even more so in Latin America, the new epicenter of COVID-19 pandemic, where converge the conditions of the disease and the social problems of a region with low-and middleincomes. We might think that having high quality Personal Protective Equipment is sufficient guarantee to be safe and the evidence shows us that it is not the only factor involved in protection or risk, as in most crises. Human factors can be determinants of protection when controlled or risk when not properly managed. Simulation centers have the capacity to participate in the construction of high-performance clinical teams that can confront safely this new enemy which threatens both patients and healthcare personnel.

\section{INTRODUCCIÓN}

Unidad de Cuidados Intensivos, 03:00

D r. D: "La enfermera me llamó, un hombre de 28 años con COVID-19 en un estado de agitación, auto-extubado. Me vestí rápidamente, entré en el cubículo, lo oxigené, le indiqué a la enfermera la secuencia de inducción rápida y lo intubé en el primer intento. Al final del proceso, las enfermeras me estaban mirando, no tenía mi equipo de protección personal (EPP) completo (traía bata larga, doble par de guantes y una mascarilla quirúrgica; no traía el respirador N95 ni las monogafas) ... No me di cuenta, nadie se dio cuenta, intenté ayudar y ahora estoy enfermo".
Sala de Emergencias, 17:30 Dra. A: "Estaba atendiendo a un paciente cuando una joven convulsionando llegó de una clínica psiquiátrica donde estaba hospitalizada para el manejo de la drogodependencia; no tenía más datos, estaba en estado de crisis, estaba saturando $80 \%$ y su frecuencia cardiaca disminuía. No había tiempo qué perder. Me puse una máscara quirúrgica, una bata y guantes tan rápido como pude, ordené pasar una carga de volumen y la secuencia de inducción rápida, la intubé, y sus signos vitales mejoraron. En la radiografía de control, vi infiltrados subpleurales bilaterales, decidí hacer una tomografía computarizada del tórax y me congelé, era compatible con COVID-19, la paciente resultó positiva ... hoy soy positiva para SARS-CoV-2". 
\| Servicio de

Anestesiología, Clínicas

Atlas. Quito, Ecuador.

^ Servicio de

Anestesiología, Hospital

Nacional de Niños

Benjamín Bloom. San

Salvador, El Salvador.

** Servicio de

Anestesiología,

Clínica Comfamiliar.

Pereira, Colombia.

\# Hospital Virtual

Valdecilla. Servicio

de Anestesiología,

Sección de Trasplante

Pulmonar, Hospital

Marqués de Valdecilla.

Santander, España.
Estas son sólo dos de las muchas historias de profesionales de la salud que han sido infectados con el nuevo SARS-CoV-2 que causa la COVID-19 (los nombres de los profesionales están ocultos por solicitud de los interesados). Esta enfermedad tiene una distribución global, es altamente transmisible y tiene desenlaces potencialmente fatales; son relatos que vivimos tan cercanos los que enseñamos a través de la simulación, pero que también somos parte del día a día de las salas de emergencias, los quirófanos y las unidades de cuidados intensivos.

Al 30 de julio de 2020, se han infectado cerca de 17 millones de personas con el nuevo coronavirus y han muerto más de 650,000 pacientes, incluido personal de salud. ${ }^{1}$ Según el director de la OMS, el 10\% de los infectados en el mundo corresponde a personal de salud, población que además sufre fatiga física y mental, combinación peligrosa para la toma de decisiones en momentos de crisis. ${ }^{2}$

Existen factores de riesgo que nos hacen más propensos al contagio y hay factores que podrían protegernos al personal de salud, como el equipo de protección personal (EPP), el dominio de habilidades no técnicas (HNT) y el manejo de la carga cognitiva (CC); en este artículo reflexionamos desde nuestra experiencia como médicos anestesiólogos e intensivistas, educadores médicos e investigadores en factores humanos y desde la revisión narrativa de la literatura sobre HNT y CC, considerando que ya hay un robusto corpus teórico disponible en cuanto a EPP.

\section{MECANISMOS DE TRANSMISIÓN}

COVID-19 se transmite principalmente por gotas y por contacto directo e indirecto, sin embargo, existen procedimientos asociados con la producción de aerosoles (intubación orotraqueal, traqueotomía, compresiones torácicas, broncoscopia, aspiración de secreciones y extubación) y recientemente se ha aceptado que es posible que también ocurra la transmisión aérea. ${ }^{3,4}$ El virus puede ingresar a través de los ojos, la nariz y la boca, por lo tanto, se debe disponer de una protección personal completa de estas estructuras, ${ }^{5,6}$ con un mensaje claro: $\sin$ piel expuesta, ya que la contaminación cutánea periocular, perioral, perinasal, del cuello y de las manos, puede entrar en contacto con la conjuntiva y mucosas descritas anteriormente y causar infección (Tabla 1).
Tabla 1: Equipo de protección personal para procedimientos generadores de aerosoles.

\begin{tabular}{ll} 
Parte del cuerpo & Protección \\
\hline Ojos & Monogafas/careta o escudo facial \\
Nariz y boca & Respirador N95 o superior \\
Manos & Doble par de guantes \\
Cuerpo & Bata larga/overol
\end{tabular}

\section{HABILIDADES NO TÉCNICAS}

Las habilidades no técnicas (HNT) son recursos personales, sociales y cognitivos que respaldan al conocimiento específico de un individuo (habilidades técnicas). HNT como el liderazgo, la comunicación, la movilización de recursos, el mantenimiento de roles, la conciencia situacional, entre otras, han sido estudiadas para el manejo de crisis. $^{7-9}$ Como hemos establecido, el mayor riesgo de contagio está en el manejo de la vía aérea, que en general, no es un asunto puramente técnico, más aún en COVID-19 donde el riesgo, además de fallar la intubación, es enfermarse, enfermar a nuestras familias y por supuesto, morir. ${ }^{10,11}$

En el primer caso, el médico nos narra una situación en la cual se vio presionado por la gravedad del paciente, que se sabía era positivo para COVID-19; sin embargo, sintió la necesidad de actuar de inmediato, esto sumado al cansancio de una jornada larga, lo llevó a la falta de consciencia situacional sobre el riesgo y el uso adecuado del EPP. Vemos también problemas en el trabajo en equipo, en el chequeo cruzado, pues su equipo tampoco notó la ausencia de protección oronasal y ocular adecuada para un procedimiento de alto riesgo de producción de aerosoles.

En el segundo caso vemos presión del contexto, problemas en la consciencia situacional y ceguera de inatención; estando en tiempo de pandemia ni la médica ni su equipo pensaron en la posibilidad de infección de la paciente.

En la Tabla 2 describimos algunas HNT y cómo abordarlas en esta pandemia.

\section{CARGA COGNITIVA}

La teoría de la carga cognitiva (CC) asume que la memoria de trabajo es bastante limitada, por 
lo que las tareas y experiencias complejas la aumentan y pueden desbordarla; esto significa una limitación para procesar la información y actuar eficientemente. La CC es de tres tipos: intrínseca, extrínseca y pertinente (Germane). La intrínseca se refiere a la dificultad de la tarea en sí (intubar, usar cajas acrílicas, trabajar en equipos pequeños, colocarse el equipo de protección personal, etcétera), la extrínseca se define como la memoria de trabajo que se ocupa de resolver situaciones que no están directamente relacionadas con la tarea (ansiedad, problemas en la comunicación debido a las máscaras, calor generado por los EPP, alteraciones en la refracción por las barreras oculares,

Tabla 2: Habilidades no técnicas para la atención de pacientes sospechosos de COVID-19.

\begin{tabular}{|c|c|}
\hline $\begin{array}{l}\text { Habilidad no } \\
\text { técnica }\end{array}$ & Recomendaciones \\
\hline $\begin{array}{l}\text { Reconozca } \\
\text { la crisis }\end{array}$ & - Dificultad respiratoria, vía aérea difícil fisiológica y/o anatómica \\
\hline $\begin{array}{l}\text { Identifique } \\
\text { al líder }\end{array}$ & $\begin{array}{l}\text { - El más experto en liderar } \\
\text { - Idealmente debe tener conocimiento de la dinámica de la } \\
\text { enfermedad y de la gestión de la vía aérea compleja }\end{array}$ \\
\hline $\begin{array}{l}\text { Asigne roles } \\
\text { al equipo }\end{array}$ & $\begin{array}{l}\text { - Minimice la cantidad de personal en sala y maximice su } \\
\text { entrenamiento } \\
\text { - Manejo de la vía aérea: el más experto } \\
\text { - Asistencia en la vía aérea: terapeuta respiratoria experta } \\
\text { - Medicamentos: enfermera experta }\end{array}$ \\
\hline $\begin{array}{l}\text { Comunicación } \\
\text { efectiva }\end{array}$ & $\begin{array}{l}\text { - Indique cabina estéril al empezar y cuando sea necesario } \\
\text { - Utilice el modelo de comunicación en asa cerrada con verificación } \\
\text { - Utilice pizarras y señales preestablecidas } \\
\text { - Coloque su nombre y rol en un lugar visible de la bata o } \\
\text { escudo facial (que no limite su función) }\end{array}$ \\
\hline $\begin{array}{l}\text { Manejo de } \\
\text { recursos }\end{array}$ & $\begin{array}{l}\text { - Use EPP completo y verificado } \\
\text { - Asegúrese de tener stock de EPP } \\
\text { - En caso de desabastecimiento tenga lineamentos claros } \\
\text { para el posible reúso } \\
\text { - Use videolaringoscopio y bougie elástico } \\
\text { - Realice los procedimientos en habitaciones con presión negativa }\end{array}$ \\
\hline $\begin{array}{l}\text { Consciencia } \\
\text { situacional }\end{array}$ & $\begin{array}{l}\text { - Haga briefing } \\
\text { - Haga donning y doffing asistido y supervisado } \\
\text { - Use listas de chequeo y haga chequeo cruzado } \\
\text { - Nombre la dinámica en alto } \\
\text { - Pausar y pensar (10 segundos para } 10 \text { minutos) } \\
\text { - Distribuya la atención } \\
\text { - Haga higiene de manos frecuente } \\
\text { - Desinfecte frecuentemente las superficies } \\
\text { - Haga debriefing }\end{array}$ \\
\hline
\end{tabular}

etcétera) y la pertinente es el esfuerzo intencional de la memoria de trabajo para realizar la tarea y transferir la información de la memoria a corto plazo a la memoria a largo plazo, ${ }^{12}$ por lo tanto, la carga intrínseca debe ser disminuida, la extrínseca minimizada y la pertinente maximizada.

En el primer caso encontramos aumento de la carga extrínseca, el contexto era agobiante, el paciente se desaturaba, las enfermeras alertaban sobre la gravedad del paciente y la necesidad de intubar rápido. La colocación del EPP no es fácil y aumenta la carga intrínseca.

En el segundo caso la carga extrínseca era alta, la médica se encontraba atendiendo a un paciente grave en la urgencia, cuando inesperadamente ingresa una paciente en falla respiratoria y convulsionando, que debía ser intubada; el equipo de respuesta no estaba listo ni informado. En ambos casos la intubación de pacientes críticamente enfermos es un reto, por lo tanto, la carga cognitiva intrínseca es alta.

En la Figura 1 planteamos algunas maneras de gestionar la CC.

\section{CONTRIBUCIÓN DE LA SIMULACIÓN CLÍNICA}

El entrenamiento en simulación clínica permite aprender conceptos y declararlos; desarrollar habilidades motoras y habilidades no técnicas, demostrarlas en la simulación y posiblemente transferir lo aprendido y desarrollado a la práctica clínica. $^{13}$

La simulación clínica con práctica deliberada es una buena manera de desarrollar habilidades para la gestión de la vía aérea, ${ }^{14}$ para entrenar en el uso de nuevas tecnologías, incluyendo los EPP más sofisticados (que son una mejor barrera, pero son difíciles de utilizar, por tanto, de mayor riesgo de contaminación durante su retiro). ${ }^{6}$

A través de la simulación clínica, se le puede ayudar a los equipos de salud a mejorar su desempeño, a hacer planeación anticipada fuera del área de atención (briefing), a utilizar listas de chequeo, a contar de rutina con un verificador para ponerse los EPP (donning), ejecutar las tareas $y$ en el retiro de los EPP (doffing). En un estudio reciente, nosotros encontramos relación entre la carga cognitiva alta y el desempeño bajo de los profesionales al usar los EPP y encontramos que el entrenamiento del donning y doffing en simulación clínica puede contribuir a bajar la carga cognitiva y aumentar la eficiencia. ${ }^{15}$ 

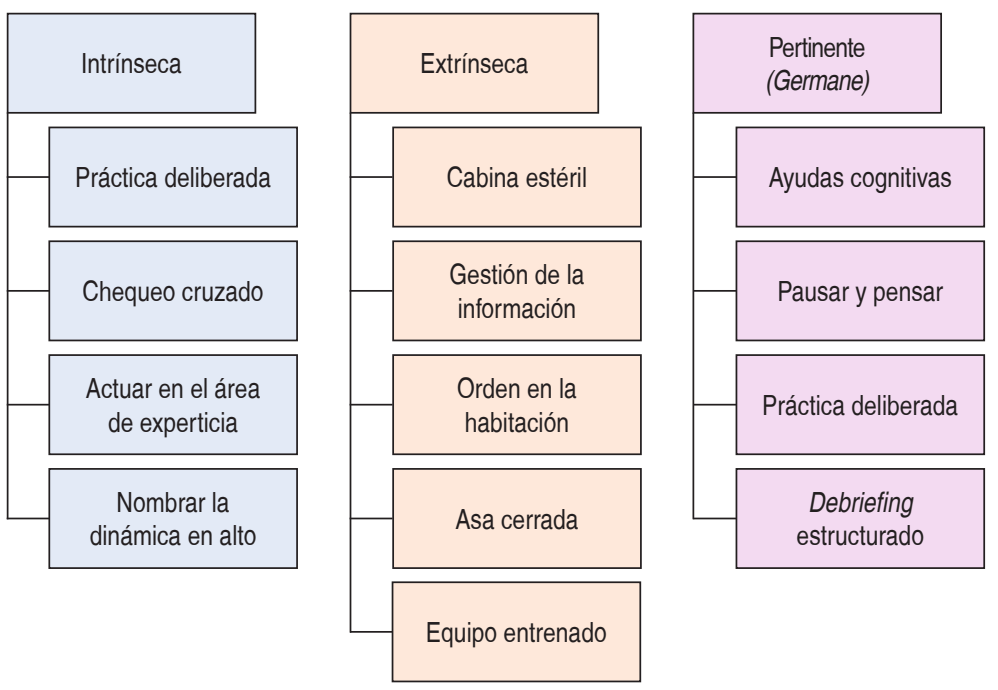

Figura 1: Estrategias para dominar la carga cognitiva.

En simulación clínica, se puede entrenar el pausar y pensar cuando la situación se empiece a salir de control, a repartir la atención, a usar listas de chequeo y a mejorar la comunicación, lo que puede ayudar a aumentar la consciencia situacional y disminuir los errores de fijación.

La simulación también permite a los profesionales de la salud aprender a tener conversaciones reflexivas al final de la atención de pacientes (debriefing clínico), ${ }^{16,17}$ lo que puede ayudar a aumentar la carga pertinente. Creemos que desde los centros de simulación tenemos mucho para aportar en esta crisis que nos invita a la resiliencia, a colaborar y a cuidarnos mutuamente. ${ }^{18}$

\section{CONCLUSIÓN}

El riesgo de contagio de COVID-19 para los trabajadores de la salud es alto, aun contando con EPP adecuados; no dependemos únicamente de las habilidades motoras, la carga cognitiva hace que lo difícil sea más difícil.

En esta pandemia se ha hecho esencial el desarrollo de habilidades no técnicas y el dominio de la carga cognitiva, los centros de simulación tienen la capacidad para formar equipos clínicos de alto rendimiento que puedan estar más seguros.

\section{REFERENCIAS}

1. WHO. Coronavirus disease 2019 - Situation Report - 191 [Internet]. World Health Organization; 2020.
Available from: https://www.who.int/docs/defaultsource/coronaviruse/situation-reports/20200729covid-19-sitrep-191. pdf?sfvrsn=2c327e9e_2

2. World Health Organization (WHO). Live media briefings on COVID-19, 17 July 2020 [Internet]. 2020. Available from: https://www.who.int/emergencies/diseases/novelcoronavirus-2019/media-resources/press-briefings

3. Cheung JC, Ho LT, Cheng JV, Cham EYK, Lam KN. Staff safety during emergency airway management for COVID-19 in Hong Kong. Lancet Respir Med. 2020; 8 (4): e19.

4. Morawska L, Milton DK. It is time to address airborne transmission of COVID-19. Clin Infect Dis. 2020; ciaa939. Available from: https://doi.org/10.1093/cid/ciaa939

5. Chu DK, Akl EA, Duda S, Solo K, Yaacoub S, Schünemann HJ, et al. Physical distancing, face masks, and eye protection to prevent person-toperson transmission of SARS-CoV-2 and COVID-19: a systematic review and meta-analysis. Lancet. 2020; 395: 1973-1987.

6. Verbeek JH, Rajamaki B, ljaz S, Sauni R, Toomey E, Blackwood B, et al. Personal protective equipment for preventing highly infectious diseases due to exposure to contaminated body fluids in healthcare staff. Cochrane database Syst Rev. 2020; 4: CD011621.

7. Flin R, Martin L, Goeters KM, Hörmann HJ, Amalberti $\mathrm{R}$, Valot C, et al. Development of the NOTECHS (nontechnical skills) system for assessing pilots' CRM skills. Human Factors Aerospace Saf. 2003; 3 (2): 95-117.

8. Gaba DM, Howard SK, Flanagan B, Smith BE, Fish KJ, Botney R. Assessment of clinical performance during simulated crises using both technical and behavioral ratings. Anesthesiology. 1998; 89 (1): 8-18.

9. Boet $S$, Bould MD, Fung $L$, et al. Transfer of learning and patient outcome in simulated crisis resource management: a systematic review. Can J Anaesth. 2014; 61 (6): 571-582.

10. Díaz-Guio Y, Salazar DF, Montoya Navarrete F, Cimadevilla-Calvo B, Díaz-Guio DA. Vía aérea difícil en el paciente crítico, mucho más que habilidades técnicas. Acta Colomb Cuid Intensivo. 2018; 18 (3): 190-198.

11. Schumacher J, Arlidge J, Dudley D, Sicinski M, Ahmad I. The impact of respiratory protective equipment on difficult airway management: a randomised, crossover, simulation study. Anaesthesia. 2020. Available from: https://onlinelibrary.wiley.com/doi/abs/10.1111/ anae. 15102

12. Sweller J. Cognitive load theory, learning difficulty, and instructional design. Learn Instr. 1994; 4 (4): 295-312.

13. Cifuentes-Gaitán MJ, González-Rojas D, RicardoZapata A, Díaz-Guio DA. Transferencia del aprendizaje de emergencias y cuidado crítico desde la simulación de alta fidelidad a la práctica clínica. Acta Colomb Cuid Intensivo [Internet]. 2020. Disponible en: https://doi. org/10.1016/j.acci.2020.06.001

14. Cook TM, El-Boghdadly K, McGuire B, McNarry AF, Patel A, Higgs A. Consensus guidelines for managing the airway in patients with COVID-19: Guidelines from the Difficult Airway Society, the Association of Anaesthetists the Intensive Care Society, the Faculty of Intensive Care Medicine and the Royal College of Anaesthetists. Anaesthesia. 2020; 75 (6): 785-799. 
15. Díaz-Guio DA, Ricardo-Zapata A, Ospina-Velez J, Gómez-Candamil G, Mora-Martinez S, RodriguezMorales AJ. Cognitive load and performance of health care professionals in donning and doffing PPE before and after a simulation-based educational intervention and its implications during the COVID-19 pandemic for biosafety. Infez Med. 2020; 28 (suppl 1): 111-117.

16. Sawyer T, Eppich W, Brett-Fleegler M, Grant V, Cheng A. More than one way to debrief. Simul Healthc. 2016; 11 (3): 209-217.

17. Maestre JM, Rudolph JW. Teorías y estilos de debriefing: el método con buen juicio como herramienta de evaluación formativa en salud. Rev Esp Cardiol. 2015; 68 (4): 282-285.

18. Díaz-Guio D, Ospina-vélez J, Ricardo-zapata A. COVID-19: una crisis que requiere medidas de formación urgentes. Simulación Clínica. 2020; 2 (1): 6-8.

Correspondencia:

Diego Andrés Díaz-Guio

Grupo de Investigación en Educación y Simulación Clínica, Centro de Simulación Clínica VitalCare. Calle 16N Núm. 14-50, Armenia, Colombia. E-mail: andres.diaz@vitalcare.co 